cerebrovascular research and clinical services, in light of the relative underfunding of those areas. They emphasize the need for primary and secondary prevention in older people, and predict a sharp increase in the rates of survival with disability which, together with an aging population, has major implications for health services.

Jim Casey

Original article Rothwell PM et al. (2005) Population-based study of event-rate, incidence, case fatality, and mortality for all acute vascular events in all arterial territories (Oxford Vascular Study). Lancet 366: 1773-1783

\section{Is PDE5 inhibition effective in pulmonary arterial hypertension?}

Phosphodiesterase type 5 (PDE5) metabolizes cyclic guanosine monophosphate, a SECOND MESSENGER that mediates the vasodilatory effects of nitric oxide. In patients with pulmonary arterial hypertension (PAH), PDE5 is upregulated, contributing to increased pulmonary vascular resistance, right ventricular failure and eventually death. Following encouraging results from preliminary studies, Galiè et al. conducted a multicenter, placebocontrolled trial investigating the efficacy and safety of sildenafil-a PDE5 inhibitor-in patients with PAH.

Patients $(n=278)$ were randomized in a 1:1:1:1 pattern to receive placebo or 20 , 40 or $80 \mathrm{mg}$ sildenafil three times daily. The primary efficacy measure was the change in exercise capacity from baseline to week 12 on 6-min walking test-an independent predictor of death in patients with idiopathic $\mathrm{PAH}$. Other measures included mean pulmonary artery pressure change and time to clinical worsening.

Patients receiving sildenafil showed increased 6-min walking distances at weeks 4,8 and 12 ( $P<0.001$ for all doses). Sildenafil significantly decreased mean pulmonary artery pressure $(P=0.04, P=0.01$ and $P<0.001$ for 20,40 and $60 \mathrm{mg}$ sildenafil, respectively). No significant difference was seen in time to clinical worsening with sildenafil compared with placebo. Most adverse events were mild to moderate in intensity for all groups and, although three of the four patients who died were in the sildenafil group, no deaths were considered to be related to study treatment.

The authors conclude that sildenafil treatment safely improves exercise capacity and decreases pulmonary artery pressure in patients with $\mathrm{PAH}$. As no dose response was found, $20 \mathrm{mg}$ of sildenafil might completely inhibit PDE5.

Rebecca Ireland

Original article Galiè N et al. (2005) Sildenafil citrate therapy for pulmonary arterial hypertension. N Engl J Med 353: $2148-2157$

\section{Fasudil as an adjunctive therapy for patients with stable angina}

As many patients with chronic stable angina remain symptomatic despite revascularization and drug therapy, there is a need for new therapeutic strategies. Several small trials of fasudil-a selective Rho-kinase inhibitor-have indicated that this treatment can reduce myocardial ischemia in patients with stable or vasospastic angina. These trials did not, however, include placebo controls, and they failed to report the use of concomitant cardiovascular drugs.

Vicari et al. have carried out a phase II, multicenter, double-blind trial designed to evaluate the safety and efficacy of fasudil in patients with stable angina who were already receiving standard medical therapy for cardiovascular disease.

Trial participants were randomized to receive either $20 \mathrm{mg}$ fasudil three times daily $(n=41)$, or placebo $(n=43)$. Dosing was gradually titrated over 8 weeks to $80 \mathrm{mg}$ three times daily. Treadmill exercise testing was used to assess patients' response to treatment after 2, 4, 6 and 8 weeks. The primary endpoint of the study was the change in total duration of exercise at peak at each time point compared with baseline.

Exercise times compared with baseline generally increased for patients treated with fasudil, and there was also a trend towards reduced myocardial ischemia with this treatment compared with placebo. Although fasudil therapy led to more-frequent skin and vascular disorders than placebo, the overall frequency of adverse events was similar in the two treatment groups.

\section{GLOSSARY}

SECOND MESSENGER

Molecule that relays signals received by cell surface receptors to intracellular target molecules; also acts to amplify the strength of a signal 